\title{
Antibody Responses 8 Months after Asymptomatic or Mild SARS-CoV-2 Infection
}

Pyoeng Gyun Choe, ${ }^{1}$ Kye-Hyung Kim, ${ }^{1}$ Chang Kyung Kang, Hyeon Jeong Suh, EunKyo Kang, Sun Young Lee, Nam Joong Kim, Jongyoun Yi, Wan Beom Park, Myoung-don Oh

Waning humoral immunity in coronavirus disease patients has raised concern over usefulness of serologic testing. We investigated antibody responses of 58 persons 8 months after asymptomatic or mildly symptomatic infection with severe acute respiratory syndrome coronavirus 2. For 3 of 4 immunoassays used, seropositivity rates were high $(69.0 \%-91.4 \%)$.

Tnfection with severe acute respiratory syndrome coronavirus 2 (SARS-CoV-2) leads to an antibody response, even in those who are completely asymptomatic. However, the initial immune response is not as strong as in patients with more severe disease, and concerns about waning immunity have been raised $(1,2)$. We evaluated the antibody responses of 58 persons in South Korea 8 months after asymptomatic or mildly symptomatic SARS-CoV-2 infection.

\section{The Study}

The eligible participants for this cross-sectional survey were persons with reverse transcription PCRconfirmed coronavirus disease (COVID-19) who had been isolated in a community treatment center (CTC) operated by Seoul National University Hospital during March 5-April 9, 2020. Isolation was in response to the COVID-19 outbreak in Daegu, South Korea (population 2.4 million), the first large outbreak outside of China, which resulted in 6,620 confirmed cases during February 18-March 24, 2020 (3). CTC admission criteria were as follows: alert, age $<65$ years, no underlying disease or well-controlled underlying disease, body temperature $<38.0^{\circ} \mathrm{C}$ with or without

Author affiliations: Seoul National University College of Medicine, Seoul, South Korea (P.G. Choe, C.K. Kang, H.J. Suh, E. Kang, S.Y. Lee, N.J. Kim, W.B. Park, M.-d. Oh); Pusan National University School of Medicine, Busan, South Korea (K.-H. Kim, J. Yi) antipyretics, and no dyspnea. During participants' CTC stay, physicians and nurses comprehensively evaluated them twice daily via video consultation. Asymptomatic persons were defined as those with body temperature $<37.5^{\circ} \mathrm{C}$ and no signs or symptoms (e.g., no subjective fever, myalgia, rhinorrhea, sore throat, cough, sputum, or chest discomfort) during the entire CTC stay; others were classified as mildly symptomatic patients (4). From all participants who provided informed consent, we collected serum samples at 8 months after infection.

We measured SARS-CoV-2-specific antibodies by using 4 commercial immunoassays: an antinucleocapsid (anti-N) panimmunoglobulin (pan-Ig) electrochemiluminescence immunoassay (ECLIA) (Elecsys AntiSARS-CoV-2; Roche Diagnostics, https://diagnostics. roche.com), an anti-N IgG ELISA (EDI Novel Coronavirus COVID-19 ELISA Kit; Epitope Diagnostics, https://www.epitopediagnostics.com), an antispike (anti-S) IgG ELISA (SCoV-2 Detect IgG ELISA; InBios International, https:/ / www.inbios.com), and an antiS1 spike subunit IgG ELISA [Anti-SARS-CoV-2 ELISA (IgG); Euroimmun, https://www.euroimmune.com]. Except for the anti-N IgG ELISA, these immunoassays were granted Emergency Use Authorization by the US Food and Drug Administration. Measurement and interpretation of results were made according to each manufacturer's instructions. For the anti-N and anti-S1 IgG ELISAs, borderline results were regarded as negative. To evaluate neutralizing activity targeting the spike receptor-binding domain, we used a surrogate virus neutralization test (sVNT) (SARS-CoV-2 Surrogate Virus Neutralization Test; GenScript, https:/ / www.genscript.com) (5). The Institutional Review Boards of Seoul National University Hospital and the Pusan National University Hospital approved the study (IRB nos. H-2009-168-1160 and H-2010-013-096).

${ }^{1}$ These first authors equally contributed to this article. 
We analyzed data from 7 participants with asymptomatic SARS-CoV-2 infection and 51 patients with mildly symptomatic COVID-19 (Table 1). Eight months after their infections, we detected anti-N pan-Ig in 53 (91.4\%), anti-N IgG in 15 (25.9\%), anti-S IgG in $50(86.2 \%)$, and anti-S1 IgG in $40(69.0 \%)(p<0.01)$ (Table 2). The sVNT found positive neutralizing activity for 31 (53.4\%). For female participants, positivity was significantly higher for anti-N IgG (40.0\% female vs. $4.3 \%$ male; $\mathrm{p}<0.01)$, anti-S IgG (94.3\% vs. $73.9 \%$; p<0.05), antiS1 IgG (82.9\% vs. $47.8 \% ; \mathrm{p}<0.01)$, and sVNT $(68.6 \%$ vs. $30.4 \%$; $p<0.01)$. Positivity by PCR for $\leq 14$ days was associated with a lower rate of positivity for anti-N pan-Ig $(50.0 \%$ for $\leq 14 \mathrm{~d}$ vs. $96.0 \%$ for $>14$ $\mathrm{d} ; \mathrm{p}<0.01$ ) (Table 2). Logistic regression analysis, for which anti-N IgG ELISA results were excluded because of exceptionally low positivity, indicated that negative results from $\geq 2$ commercial immunoassays were significantly associated with positivity by PCR for $\leq 14$ days after adjustment for sex (adjusted odds ratio 11.49; 95\% CI 1.45-90.79; $\mathrm{p}=$ 0.02) (Appendix, https://wwwnc.cdc.gov/EID/ article/27/3/20-4543-App1.pdf).

\section{Conclusions}

Knowledge of the longevity of humoral immunity to SARS-CoV-2 is essential for predicting herd immunity and interpreting seroepidemiologic data. Recent studies showed that the antibody titers of patients with mild SARS-CoV-2 infection declined more quickly than those reported for SARS-CoV patients (6), and waning immunity was confirmed 5 months after infection (7). Concern about the usefulness of population-based seroprevalence studies has been raised because rapidly waning immunity may lead to a substantial number of false-negative immunoassay results (2). However, in this study, we confirmed that rates of antibody positivity according to 3 commercial kits was still high at 8 months after infection, even in asymptomatic or mildly symptomatic participants (69.0\%-91.4\%). Rates differed according to immunoassay methods or manufacturers, thereby explaining differences in rates between the studies $(2,8)$. A previous study argued that among asymptomatic persons who had been antibody positive early in the infection, $40 \%$ became antibody negative in $2-3$ months, even when tested by chemiluminescence immunoassay (CLIA) (2); however, their results are in stark contrast to ours, which may have resulted from variations in the characteristics of CLIA products from different manufacturers. In a systematic review and meta-analysis, pooled sensitivity was $97.8 \%$ with CLIA in contrast to $84.3 \%$ with ELISA (9). In a head-to-head benchmark comparison study, anti-N pan-Ig ECLIA showed $97.2 \%$ sensitivity and $99.8 \%$ specificity (10). In the previous studies, CLIA showed high sensitivity and specificity for recent or past SARS-CoV-2 infection. Therefore, our results show that a serosurvey is useful even 8 months after an outbreak if an appropriate binding immuno-

\begin{tabular}{|c|c|c|}
\hline Characteristic & Asymptomatic & Mildly symptomatic \\
\hline Total no. persons & 7 & 51 \\
\hline \multicolumn{3}{|l|}{ Sex, no. (\%) } \\
\hline $\begin{array}{l}\mathrm{M} \\
\mathrm{F}\end{array}$ & $\begin{array}{l}5(71.4) \\
2(28.6)\end{array}$ & $\begin{array}{l}18(35.3) \\
33(64.7)\end{array}$ \\
\hline Age, y, median (IQR) & $25(21-26)$ & $26(22-40)$ \\
\hline Underlying disease, no. (\%)† & 0 & $3(5.9)$ \\
\hline \multicolumn{3}{|l|}{ Smoking status } \\
\hline Smoker & 0 & 0 \\
\hline Ex-smoker & $1(14.3)$ & $2(3.9)$ \\
\hline Nonsmoker & $6(85.7)$ & $49(96.1)$ \\
\hline \multicolumn{3}{|l|}{ Signs/symptoms, no. (\%) } \\
\hline Febrile/chilling sense & NA & $8(15.7)$ \\
\hline Myalgia & NA & $5(9.8)$ \\
\hline Headache & NA & $13(25.5)$ \\
\hline Cough & NA & $20(39.2)$ \\
\hline Sputum & NA & $33(64.7)$ \\
\hline Rhinorrhea & NA & $25(49.0)$ \\
\hline Sore throat & NA & $3(5.9)$ \\
\hline Chest discomfort/dyspnea & NA & $4(7.8)$ \\
\hline Duration of PCR positivity, $\mathrm{d}$, median (IQR) & $29(25-34)$ & $24(19-34)$ \\
\hline Days from symptom onset to blood sampling, median (IQR)‡ & $231(231-233)$ & $234(231-234)$ \\
\hline
\end{tabular}


Table 2. Positivity of antibodies to severe acute respiratory syndrome coronavirus 2 in 58 asymptomatic or mildly symptomatic patients at $8 \mathrm{mo}$ after infection, South Korea*

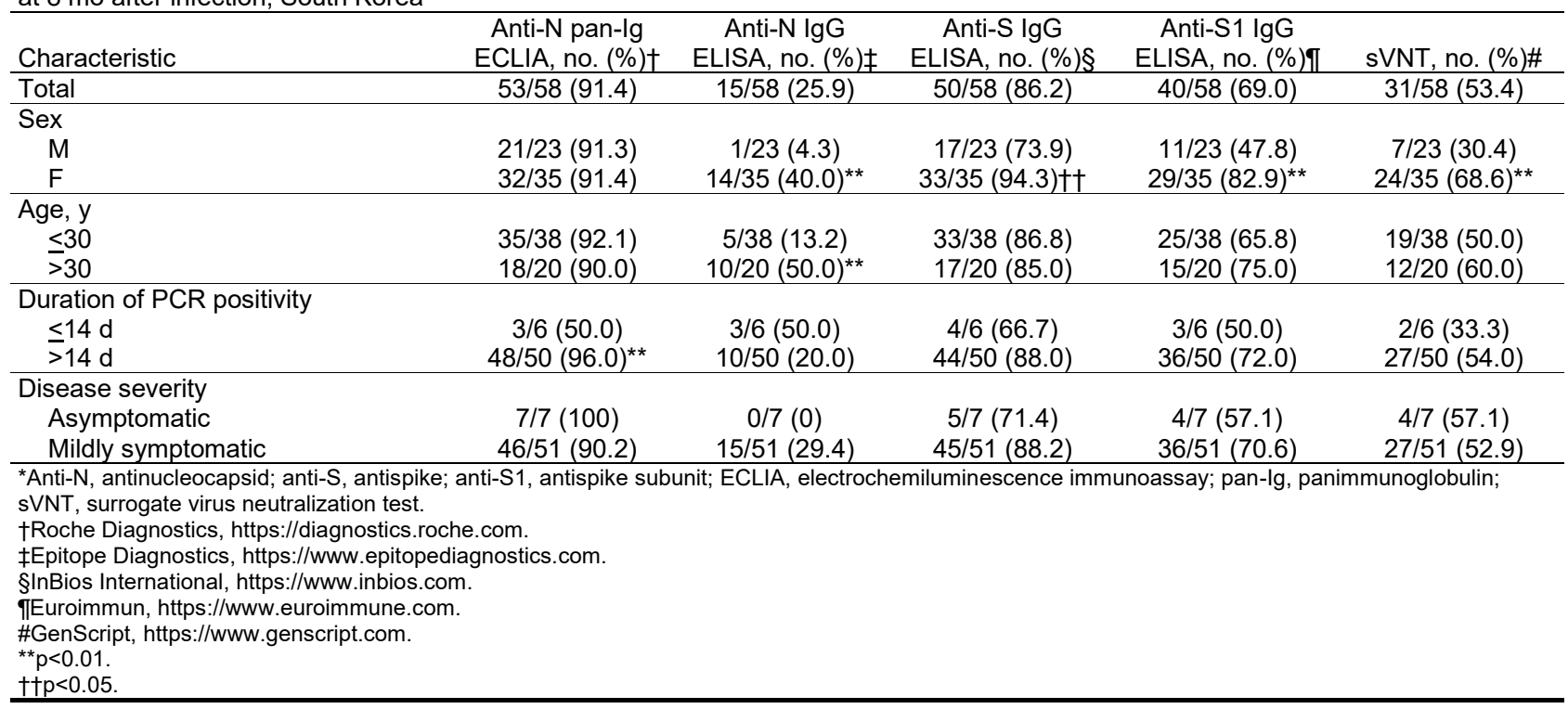

assay format like an anti-N pan-Ig ECLIA is used. A serosurvey that uses a binding immunoassay can determine the infected proportion of the population and also the proportion of infections detected by PCR, thus enabling inference of the infectionfatality ratio rather than just the case-fatality ratio; however, it cannot accurately assess population immunity because it is not a functional immunoassay for detecting neutralizing activity.

Neutralizing activity, a functional aspect of antibodies, is essential for protection from reinfection and screening potential convalescent plasma therapy donors (8). In our study, neutralizing activity was detected in $53.4 \%$ of asymptomatic or mildly symptomatic participants after 8 months of infection, which was considerably lower than the rate of positivity detected by binding immunoassays such as ECLIA or ELISAs. This finding is not surprising because neutralizing activity is affected by various factors, including the antigen specificity and the amount of existing antibody. However, confirming sVNT results by conventional VNT might be needed, although the reported specificity $(100 \%)$ and sensitivity $(98 \%-98.9 \%)$ of sVNT showed good correlation with conventional VNT (5). A recently published study of convalescent plasma therapy found detectable neutralizing antibodies in $63.6 \%$ persons a median of 41 days after PCR-confirmed diagnosis of mild COVID-19 (11).

According to our study, prolonged duration of virus shedding is associated with long-term antibody positivity in patients with mild COVID-19, which aligns with previous findings of higher IgG levels during weeks $4-8$ in those in the prolonged virus shedding group (12). Factors associated with prolonged virus shedding include male sex, old age, severe illness at admission, and invasive mechanical ventilation (13). Our findings suggest that the duration of virus shedding reflects the amount of humoral immune stimulation, even in asymptomatic or mildly symptomatic persons with COVID-19.

One limitation of our study was the relatively small sample size and the predominantly young population, which lessen generalization of the results. Also, because of the cross-sectional design, we could not obtain baseline or longitudinal serum samples. For the 7 asymptomatic participants in our study, we evaluated antibody responses at 2 and 5 months after infection; $5 / 7(71 \%)$ had positive ELISA results at 2 months after infection, $4 / 7(57.1 \%)$ had positive ELISA results at 5 months after infection, and all had neutralizing antibodies at 2 and 5 months after infection $(1,7)$. Last, we could not assess the individual possibilities of reexposure or reinfection. However, it is unlikely that humoral immunity was boosted because in Daegu, where the study participants reside, during April-October 2020, the daily incidence rate for COVID-19 was $<0.5$ cases/100,000 population (14). In conclusion, despite concerns of waning immunity, appropriate immunoassays can detect antibodies against SARS-CoV-2 at 8 months after infection in most asymptomatic or mildly symptomatic persons. 


\section{Acknowledgments}

We thank Kyung Sook Ahn for administrative support and Areum Jo, Su Jin Choi, and Mee Kyung Ko for technical support.

This work was supported by a 2-year research grant from Pusan National University. The funding agencies had no role in the design and conduct of the study; collection, management, analysis, and interpretation of the data; preparation, review, or approval of the manuscript; and decision to submit the manuscript for publication.

\section{About the Author}

Dr. Choe is a clinical scientist at Seoul National University Hospital. His research interests focus on preventing healthcare-associated infection and responding to emerging infectious diseases.

\section{References}

1. Choe PG, Kang CK, Suh HJ, Jung J, Kang E, Lee SY, et al. Antibody responses to SARS-CoV-2 at 8 weeks postinfection in asymptomatic patients. Emerg Infect Dis. 2020;26:2484-7. https:/ / doi.org/10.3201/eid2610.202211

2. Long QX, Tang XJ, Shi QL, Li Q, Deng HJ, Yuan J, et al. Clinical and immunological assessment of asymptomatic SARS-CoV-2 infections. Nat Med. 2020;26:1200-4. https:/ / doi.org/10.1038/s41591-020-0965-6

3. Kang E, Lee SY, Jung H, Kim MS, Cho B, Kim YS. Operating protocols of a community treatment center for isolation of patients with coronavirus disease, South Korea. Emerg Infect Dis. 2020;26:2329-37. https:/ / doi.org/10.3201/ eid2610.201460

4. Choe PG, Kang EK, Lee SY, Oh B, Im D, Lee HY, et al. Selecting coronavirus disease 2019 patients with negligible risk of progression: early experience from non-hospital isolation facility in Korea. Korean J Intern Med. 2020;35:76570. https:/ / doi.org/10.3904/ kjim.2020.159

5. Tan CW, Chia WN, Qin X, Liu P, Chen MI, Tiu C, et al. A SARS-CoV-2 surrogate virus neutralization test based on antibody-mediated blockage of ACE2-spike protein-protein interaction. Nat Biotechnol. 2020;38:1073-8. https:/ / doi.org/ 10.1038/s41587-020-0631-z
6. Ibarrondo FJ, Fulcher JA, Goodman-Meza D, Elliott J, Hofmann C, Hausner MA, et al. Rapid decay of anti-SARSCoV-2 antibodies in persons with mild Covid-19. N Engl J Med. 2020;383:1085-7. https://doi.org/10.1056/NEJMc2025179

7. Choe PG, Kang CK, Suh HJ, Jung J, Song KH, Bang JH, et al. Waning antibody responses in asymptomatic and symptomatic SARS-CoV-2 infection. Emerg Infect Dis. 2020;27. Epub 2020 Oct 13.

8. Wajnberg A, Amanat F, Firpo A, Altman DR, Bailey MJ, Mansour M, et al. Robust neutralizing antibodies to SARS-CoV-2 infection persist for months. Science. 2020;370:1227-30. https:/ / doi.org/10.1126/science.abd7728

9. Lisboa Bastos M, Tavaziva G, Abidi SK, Campbell JR, Haraoui LP, Johnston JC, et al. Diagnostic accuracy of s erological tests for covid-19: systematic review and meta-analysis. BMJ. 2020;370:m2516. https://doi.org/ 10.1136/bmj.m2516

10. Ainsworth M, Andersson M, Auckland K, Baillie JK, Barnes E, Beer S, et al.; National SARS-CoV-2 Serology Assay Evaluation Group. Performance characteristics of five immunoassays for SARS-CoV-2: a head-to-head benchmark comparison. Lancet Infect Dis. 2020;20:1390-400. https://doi.org/10.1016/S1473-3099(20)30634-4

11. Agarwal A, Mukherjee A, Kumar G, Chatterjee P, Bhatnagar T, Malhotra P; PLACID Trial Collaborators. Convalescent plasma in the management of moderate covid-19 in adults in India: open label phase II multicentre randomised controlled trial (PLACID Trial). BMJ. 2020;371:m3939. https://doi.org/10.1136/bmj.m3939

12. Jin CC, Zhu L, Gao C, Zhang S. Correlation between viral RNA shedding and serum antibodies in individuals with coronavirus disease 2019. Clin Microbiol Infect. 2020; S1198-743X(20)30299-8.

13. Xu K, Chen Y, Yuan J, Yi P, Ding C, Wu W, et al. Factors associated with prolonged viral RNA shedding in patients with coronavirus disease 2019 (COVID-19). Clin Infect Dis. 2020;71:799-806. https://doi.org/10.1093/cid/ciaa351

14. Ministry of Health and Welfare. Coronavirus disease-19, Republic of Korea [cited 2020 Nov 5]. http:/ /ncov.mohw.go.kr/en

Address for correspondence: Jongyoun Yi, Department of Laboratory Medicine, Pusan National University School of Medicine, 179 Gudeok-ro, Seo-gu, Busan, 49241, South Korea; email: socioliberal@yahoo.co.kr; Wan Beom Park, Department of Internal Medicine, Seoul National University College of Medicine, 103 Daehak-ro, Jongro-gu, Seoul, 03080, South Korea; email: wbpark1@snu.ac.kr 